\title{
A Política De Atenção Oncológica Num Município Da Região Dos Lagos Do Rio De Janeiro
}

The Oncological Attention Policy In A Municipality Of The Lagos Region Of Rio De Janeiro

\author{
Fernanda Arnaudin Soares Silva ${ }^{1}$ \\ Sérgio Nunes de Sousa Porto ${ }^{2}$ \\ Cristiano Bertolossi Marta ${ }^{3}$ \\ Priscilla Oliveira da Silva ${ }^{4}$ \\ Ronilson Rocha Gonçalves 5
}

Antônio Augusto de Freitas Peregrino ${ }^{6}$

\footnotetext{
1. Email: fernanda_arnaudin@hotmail.com. Enfermeira residente pela Universidade Federal do Estado do Rio de Janeiro, pós graduada em Enfermagem em Terapia Intensiva pelo Instituto Israelita de Ensino e Pesquisa Albert Einstein, formada pela Universidade Veiga de Almeida - Cabo Frio / RJ. Hospital Universitário Gaffrée e Guinle - UNIRIO

2. Email: snsporto@hotmail.com. Mestre em Ciências da Saúde. Professora Assistente do Curso de Graduação em Enfermagem da Universidade Veiga de Almeida

3. Email: cristianobertol@gmail.com. Pós doutorado em Enfermagem com ênfase em Avaliação de Tecnologias em Saúde. Professor Adjunto da Faculdade de Enfermagem da UERJ. Diretor de Pesquisa da Universidade Veiga de Almeida. Laboratório de Avaliação Econômica e de Tecnologias em Saúde (LAETS) da UNIRIO.

4. Email: priscillaods@yahoo.com.br. Mestre em Saúde Coletiva. Professora Assistente do Curso de Graduação em Enfermagem da Universidade Veiga de Almeida

5. Email: ronilsonprof@gmail.com. Doutor em Enfermagem. Professor Adjunto da Faculdade de Enfermagem da UERJ.

6. Email: antonio.peregrino@gmail.com. Pós doutorado em Enfermagem. Laboratório de Ciências Radiológicas. Universidade do Estado do Rio de Janeiro. Laboratório de Avaliação Econômica e de Tecnologias em Saúde (LAETS) da UNIRIO.
} 


\title{
Resumo
}

O câncer atualmente representa um problema de saúde pública com incidência crescente no Brasil, assim como no mundo. Objetivo: analisar a qualidade da atenção oncológica a partir da visão dos usuários do SUS que já receberam algum tipo de assistência. Foram revisados tópicos sobre envelhecimento, oncopatias, legislação e ações de controle. Metodologia: Trata-se de uma pesquisa de campo, com abordagem quantitativa, descritiva e exploratória, a coleta de dados foi realizada através da aplicação de um formulário. Os participantes dessa pesquisa foram pacientes oncológicos, maiores de 18 anos, tratados pelo SUS e munícipes de Cabo Frio, cenário da pesquisa foi uma UNACON, localizada no município já citado. Resultados: Em face dos resultados obtidos, esta pesquisa identificou algumas lacunas na assistência a partir do relato dos usuários do sistema. Os resultados foram dispostos em cinco tópicos que compõem as dimensões abrangidas pela PNPCC: Promoção da saúde, Prevenção, Diagnóstico precoce, Tratamento oportuno e Cuidados paliativos. Conclusão: Percebe-se que, assim como no país, o município não consegue suprir a carência de toda clientela de forma equitativa e tempestiva. Devido ao aumento progressivo de acometidos pela doença, as estruturas de atendimento terciário deveriam crescer similarmente para que pudessem garantir os direitos da população, de atendimento integral e devida oferta terapêutica, assim como preconizado pela política oncológica.

Palavras-chave: Oncologia; Política de Saúde; Gestão em Saúde.

\begin{abstract}
Cancer currently represents a public health problem with a growing incidence in Brazil, as well as in the world. Objective: to analyze the quality of cancer care based on the vision of SUS users who have already received some kind of assistance. Topics on aging, oncology, legislation and control actions were reviewed. Methodology: This is a field research, with a quantitative, descriptive and exploratory approach, data collection was performed through the application of a form. The participants of this research were cancer patients, over 18 years old, treated by the SUS and Cabo Frio residents, the research scenario was a UNACON, located in the municipality already mentioned. Results: In the face of the results obtained, this research identified some gaps in the assistance from the system users' reports. The results were presented in five topics that comprise the dimensions covered by the PNPCC: Health Promotion, Prevention, Early Diagnosis, Timely Treatment and Hospice Care. Conclusion: It can be seen that, just as in the country, the municipality can not meet the needs of all clients in an equitable and timely manner. Due to the progressive increase of those affected by the disease, the tertiary care structures should grow similarly so that they could guarantee the population's rights, full care and due therapeutic offer, as recommended by the cancer policy.
\end{abstract}

Keywords: Oncology; Health Policy; Health Management. 


\section{Introdução}

Sabe-se que a longevidade populacional vem aumentando para níveis de idade cada vez maiores e, que associados aos hábitos de vida irregulares e não saudáveis, vem influenciando no aumento progressivo no número de pessoas acometidas pelos mais variados tipos de doenças, entre as quais as neoplasias $^{(1)}$. Maiores impactos destas são observados nos países em desenvolvimento não só pela associação do câncer (CA) com a pobreza, mas também pela baixa quantidade de recursos disponibilizados para seus diagnósticos precoces e tratamentos, que em geral são de elevados custos $^{(2)}$.

É importante lembrar que CA é o termo usado para denominar um amplo grupo de doenças que se caracterizam por apresentar multiplicação celular rápida e desordenada, ocasionando a formação de tumores malignos. Estes tipos de neoplasias tendem a ser agressivas e incontroláveis, fatores que contribuem para altos índices de mortalidade mundial(3).

O CA atualmente representa um problema de saúde pública, e sua incidência vêm crescendo cada vez mais no mundo inteiro, assim como no Brasil. O Instituto Nacional do Câncer (INCA) aponta a ocorrência de cerca de 576 mil novos casos no ano de 2014, estimativa que também será válida para 2015. Esses dados reforçam o tamanho do problema desta enfermidade no país ${ }^{(4)}$.

Cabe destacar que seu controle está diretamente ligado a prevenção e detecção precoce. É importante lembrar que a assistência prestada à população perpassa por todos os níveis de atenção, contemplando ações de promoção da saúde, prevenção, detecção precoce, tratamento oportuno e cuidados paliativos $^{(5)}$.

É importante ressaltar que os cuidados paliativos se centram na qualidade e não na duração da vida. Oferecem assistência humana e compassiva para as pessoas nas últimas fases de uma doença incurável para que possam viver o mais confortavelmente possível.

Sendo assim, foi fundamental a implantação de um programa nacional como estratégia de controle do câncer. Atualmente, a Política Nacional para Prevenção e Controle do Câncer (PNPCC) está estabelecida pela Portaria n.o 874, de 16 de maio de 2013 com o intuito de melhorar a qualidade na assistência e, consequentemente, diminuir o perfil de morbimortalidade ainda alto no país $^{(5)}$; e a portaria SAS/MS n.o 741, de 19 de dezembro de 2005, estabelece normas e 
critérios para a habilitação dos serviços de Alta Complexidade na Rede de Atenção Oncológica(6).

Como estratégia de controle da doença, foi implantado em todo o país Centros de Assistência de Alta Complexidade em Oncologia (CACONs) e Unidades de Assistência de Alta Complexidade em Oncologia (UNACONs) que têm o intuito de ampliar a cobertura de atendimento e a finalidade de proporcionar aos pacientes um diagnóstico definitivo, determinar estadiamento da doença e ofertar tratamento com qualidade de acordo com as condutas estabelecidas $(5,6)$.

$$
\text { O município de Cabo Frio - RJ conta }
$$
com a implantação de uma UNACON desde 2008, que dispõe da capacidade de atendimento aos pacientes moradores da Região dos Lagos, acometidos pelos tipos de câncer mais prevalentes do país ${ }^{(7)}$. Dessa forma, o Governo Federal disponibiliza o repasse do teto orçamentário da Média e Alta Complexidade (MAC) para essa unidade, assegurando o direito a assistência completa dos pacientes usuários do SUS ${ }^{(8)}$.

Por tanto, este estudo tem como objeto a implementação da Política Nacional para Prevenção e Controle do Câncer no município de Cabo Frio - RJ. Neste contexto, tem como objetivo analisar a qualidade da atenção oncológica, a partir da visão dos usuários do Sistema Único de Saúde (SUS) que já receberam algum tipo de assistência.

Este estudo busca contribuir como tema para debates e discussões com a finalidade de melhorar o processo de gestão da Política Oncológica da Cidade de Cabo Frio e, consequentemente, prestar um melhor atendimento oncológico aos pacientes tratados na Rede Pública desta localidade. Além de contribuir com a comunidade científica para realização de estudos futuros.

\section{Método}

Para realização deste artigo foi utilizado o método de pesquisa de campo com abordagem quantitativa, descritiva e exploratória. Tem característica de pesquisa de campo quantitativa, pois após a coleta de relatos dos usuários do sistema realizou-se a análise da qualidade da PNPCC no município de Cabo Frio - RJ, posteriormente, as evidências traduziram-se em números.

A pesquisa de campo é utilizada como método para obter informações e conhecimentos que respondam ou comprovem hipóteses, além de esclarecer possíveis fenômenos ou relação entre eles ${ }^{(9)}$.

Possui perfil exploratório-descritivo, pois abordou os pacientes oncológicos que receberam algum tipo de tratamento do sistema, para saber se a vivência destes vai de encontro a ações preconizadas pela PNPCC, as questões abordadas foram analisadas sem 
interferência do pesquisador e, a partir deste ponto, possíveis lacunas puderam ser identificadas.

O cenário do estudo foi uma UNACON, localizada no município de Cabo Frio - RJ. Por ser a unidade de referência em tratamento de alta complexidade, entende-se que os pacientes tratados lá já passaram por todos os níveis de atenção. Esta conta com três consultórios, destinados a dois médicos e um para enfermeiro, uma ampla sala destinada ao tratamento quimioterápico e sala de espera. Participaram desse estudo, pacientes com diagnóstico confirmado de oncopatias, com idade maior que 18 anos, tratados pelo SUS e munícipes de Cabo Frio. Foram excluídos da pesquisa os sujeitos que não se disponibilizaram a participar da pesquisa, que tiveram que interromper a participação por alguma descompensação física ou psicológica e que estavam aguardando a algum resultado de confirmação de seu diagnóstico.

A pesquisa de campo foi embasada na legalidade na Resolução n. ${ }^{\circ} 466$ do Conselho Nacional da Saúde (CNS), de 12 de dezembro de 2012 que autoriza o estudo com seres humanos em território brasileiro, para a entrada no campo foi realizado contato prévio e apenas após a autorização documentada da coordenação responsável se iniciou a pesquisa em campo $^{(9)}$. 0 projeto foi enviado ao Comitê de Ética e Pesquisa (CEP) da Universidade Veiga de Almeida sendo aprovado sob o parecer n.ㅇ 2.229.943. Adicionalmente, foi utilizado o Termo de Consentimento Livre e Esclarecido (TCLE) junto aos pacientes entrevistados e a proteção das respectivas imagens com utilização de nomes fictícios.

A princípio foram coletados dados pertencentes às características dos participantes como sexo, idade, escolaridade, entre outros. Já a investigação se deu por uma entrevista estruturada com a utilização de um formulário contendo 17 perguntas fechadas. Neste, o participante foi questionado quanto às variáveis contempladas pela PNPCC (ações de promoção, prevenção, detecção precoce, tratamento oportuno e cuidados paliativos). As respostas obtidas relacionam-se à vivência dos pacientes durante o período que receberam assistência nos níveis de atenção básica, secundária e alta-complexidade.

Foram entrevistados 36 participantes, todas as quartas-feiras, pois é o dia de maior fluxo de atendimento aos pacientes do SUS, durante o mês de setembro de 2017. Ao decorrer dos dias da pesquisa, a equipe tornou-se mais receptiva e apenas seis sujeitos dentro dos critérios de inclusão recusaram-se responder o formulário.

Os resultados foram apresentados conforme o método estatístico descritivo, pois transformou a vivência dos pacientes em números que traduziram a funcionalidade da PNPCC, convertendo esses dados em 
percentual e apresentando em tabelas e gráficos.

Desta forma, a avaliação da qualidade da PNPCC ofertada na cidade de Cabo Frio - RJ realizou-se por munícipes usuários do sistema.

\section{Resultados}

Ao traçar um perfil sócio epidemiológico dos pacientes oncológicos moradores do primeiro (81\%) e segundo (19\%) distritos de Cabo Frio - RJ, com 36 participantes apresentando idades entre 40 a 82 anos, sendo que $92 \%$ apresentaram idade igual ou superior a 50 anos, cabe destacar um maior predomínio do sexo feminino (67\%).

Quanto à escolaridade, constatou-se que a soma dos participantes com formação até nível médio é de $83 \%$, seguidos de $11 \%$ com nível superior e $6 \%$ de analfabetos. Sabese que a escolaridade está diretamente ligada a fatores socioeconômicos.

Outro fator importante a se destacar é a incidência do câncer por gênero. Nesta pesquisa, $50 \%$ das mulheres foi diagnosticada com CA de mama, seguida de CA de intestino, ovário, endométrio e estomago com $8 \%$ respectivamente, além dos tipos de CA de fígado, útero, esôfago e coluna que apresentam 4\% cada. Já nos homens a predominância é nos casos de CA de próstata (42\%), seguido de CA de intestino (25\%) e de
CA de cabeça, estômago, pulmão e osso com $4 \%$ cada. Os valores ressaltados estão de acordo com os níveis nacionais ${ }^{(4-10)}$.

Para analisar os dados, a discussão foi dividida em cinco tópicos que compõem as dimensões abrangidas pela Política Nacional para Prevenção e Controle do Câncer (PNPCC): promoção da saúde, prevenção, diagnóstico precoce, tratamento oportuno e cuidados paliativos.

\section{Promoção da saúde}

Neste tópico, os participantes foram questionados sobre o conhecimento relativo aos fatores de risco para o desenvolvimento do câncer e seus hábitos de vida antes da doença e os resultados podem ser observados na Tabela 1. 
Tabela 1. Relação entre o conhecimento populacional sobre os fatores de risco e o autocuidado. Cabo Frio, RJ, Brasil, 2017.

\begin{tabular}{|c|c|c|c|c|c|}
\hline Fatores de Risco & $\begin{array}{c}\text { Exposição } \\
N\end{array}$ & Exposição $(\%)$ & $\begin{array}{c}\text { Conhecimento } \\
\text { N }\end{array}$ & $\begin{array}{c}\text { Conhecimento } \\
|(\%)|\end{array}$ & TOTAL \\
\hline Alimentação inadequada & 11 & 31 & 17 & 47 & \\
\hline Sedentarismo & 24 & 67 & 21 & 58 & \\
\hline Exposição solar sem proteção & 29 & 81 & 28 & 78 & \\
\hline Tabagismo & 15 & 42 & 35 & 97 & \\
\hline Consumo de Álcool inadequado & 1 & 3 & 24 & 67 & \\
\hline Obesidade & $\cdot$ & $\cdot$ & 17 & 47 & \\
\hline Sexo sem preservativo & . & . & 25 & 69 & \\
\hline Agrotóxicos & . & . & 31 & 86 & \\
\hline Vacinação irregular & 11 & 33 & . & . & \\
\hline Prevenção & & $\operatorname{sim}$ & & Não & \\
\hline Recebeu palestra preventiva & 9 & 25 & 27 & 75 & $36(100 \%)$ \\
\hline Buscava atendimento médico & 24 & 67 & 11 & 33 & $36(100 \%)$ \\
\hline
\end{tabular}

Realizava exames com

$\begin{array}{llllll}\text { regularidade } & 24 & 67 & 11 & 33 & 36(100 \%)\end{array}$

Nota: $(\mathrm{N}=36)$

Fonte: dados da pesquisa

É perceptível, que boa parte dos entrevistados relatou conhecer os fatores predisponentes para o câncer e, curiosamente, alguns mantêm o comportamento de risco. A razão desse conhecimento pode estar relacionada à implantação de políticas públicas de promoção a saúde que dão prioridade a ações, como: alimentação saudável, atividades físicas e prevenção ao uso do tabaco e álcool(11).

\section{Prevenção}

Sobre essa temática, apenas uma pequena parcela (25\%) dos participantes afirmou já ter recebido palestra preventiva contra o câncer, destes, a maior parte (44\%) informou como local de oferta ambiente de trabalho, seguido de unidades de saúde (33\%) e outros (22\%).

Em geral, a maioria (67\%) buscava atendimento médico e realizava exames rotineiramente. Estes, quando questionados sobre a frequência que buscavam atendimento médico, 39\% respondeu anualmente, $17 \%$ semestralmente e $11 \%$ relatou outra frequência, entre mensal até bianual. Observou-se que 33\% não buscou atendimento ambulatorial e, consequentemente, não realiza exames de rotina (Tabela 1).

A maioria dos participantes afirmou nunca ter recebido palestra de prevenção do câncer, entretanto, a chave para diminuir a incidência do câncer é a educação da população, uma ação básica que esclarece sobre o desenvolvimento da doença e a possibilidade de prevenção dela. A Estratégia de Saúde da Família (ESF) é a porta de entrada do serviço de saúde e também o local mais propício para desenvolver atividades educativas $^{(12)}$.

\section{Diagnóstico precoce}

Neste item, os integrantes da pesquisa relataram os pontos de dificuldades para a descoberta da doença, como pode ser visualizado na Tabela 2.

Tabela 2. Principais dificuldades enfrentadas pelos usuários SUS no diagnóstico do câncer.

Cabo Frio, RJ, Brasil, 2017.

Nota: $(\mathrm{N}=36)$ 


\begin{tabular}{|c|c|c|c|c|c|}
\hline Dificuldades & $\sin (\mathbb{N})$ & $\operatorname{sim}|y|$ & Nầo (N) & Nâa $|y|$ & TOTAL \\
\hline Consulta Mélica & 5 & 14 & 31 & 86 & $36(1008)$ \\
\hline Exames Diagniositicos & 11 & 33 & 25 & 67 & $36(1008)$ \\
\hline Biópsia & 8 & 22 & . & . & \\
\hline Ressonoancia Magneticta & 4 & 11 & . & . & \\
\hline Endoscopia & 2 & 6 & . & . & \\
\hline Uttrassonogeralia & 2 & 6 & . & . & \\
\hline Outros & 4 & 11 & . & . & \\
\hline \multirow{3}{*}{ Custos dos Exames } & Conta & Conta & $\operatorname{sus}(N)$ & $\operatorname{sus}\left|x_{0}\right|$ & \\
\hline & $\begin{array}{l}\text { Propila } \\
|N|\end{array}$ & Proppria|yo| & & & \\
\hline & 14 & 39 & 22 & 61 & \\
\hline
\end{tabular}

Fonte: dados da pesquisa

Observa-se que a maioria dos entrevistados não relatou dificuldades para o encaminhamento à consulta médica específica e nem quanto à realização de exames diagnósticos, além disso, confirmam que o custeio destes exames foi realizado pelo SUS, relativo aos que apresentaram dificuldades pode-se justificar pelo aumento progressivo de casos da doença( ${ }^{(4)}$.

\section{Tratamento oportuno}

Nesta dimensão, os participantes relataram os tratamentos realizados $\mathrm{e}$ as principais dificuldades enfrentadas para realizálos, tais dados podem ser notados na Tabela 3.
Tabela 3. Oferta de tratamento assistencial

oncológico. Cabo Frio, RJ, Brasil, 2017.

\begin{tabular}{|c|c|c|c|c|c|}
\hline Diagnóstico x Tratamento & $\begin{array}{l}\text { Participantes } \\
\text { (N) }\end{array}$ & \multicolumn{3}{|c|}{$\begin{array}{c}\text { Participantes } \\
(\%)\end{array}$} & TOTAL \\
\hline Até60 dias & 20 & & & 56 & \\
\hline Acima de 60 dias antes da resolução & 5 & & & 14 & \\
\hline Acima de 60 dias após resolução & 5 & & & 14 & \\
\hline Não souberam responder & 6 & & & 6 & \\
\hline Principais Tratamentos & . & & & - & \\
\hline Quimioterapia & 30 & & & 3 & \\
\hline Radioterapia & 25 & & & 9 & \\
\hline Cirurgia & 14 & & & 9 & \\
\hline \multirow{3}{*}{ Dificuldades no tratamento } & $\operatorname{sim}(\mathbb{N})$ & Sim & Não(N) & Não $(\%)$ & TOTAL \\
\hline & & $(\%)$ & & & \\
\hline & 7 & 19 & 29 & 81 & $36(100 \%)$ \\
\hline Radioterapia & 6 & 17 & . & . & . \\
\hline Cirurgia & 1 & 2 & . & . & . \\
\hline Uso Medicação em casa & 11 & 31 & 25 & 69 & $36(100 \%)$ \\
\hline Comprados & 3 & 27 & & - & \\
\hline
\end{tabular}

\begin{tabular}{cccccc} 
SUS & 8 & 73 & & \\
TOTAL & 11 & 100 & & $\cdot$ & \\
Cuidados Paliativos & & & & & \\
Vistata Domicliar & 3 & 8 & 33 & 92 & 36 (100\%) \\
Acompanhamento Psicológico & 3 & 8 & 33 & 92 & 36 (100\%) \\
Oferta de suporte emocional a familia & 9 & 25 & 27 & 75 & 36 (100\%) \\
\hline
\end{tabular}

Nota: $(\mathrm{N}=36)$

Fonte: dados da pesquisa

Observa-se que a grande maioria dos participantes conseguiu ser atendido em até 60 dias, fato que corrobora com as leis nacionais sobre a temática.

Dentre toda a oferta de tratamentos disponibilizados pelo SUS, foi possível observar que os mais utilizados são quimioterapia e radioterapia. E as principais dificuldades informadas para o início dos tratamentos estão ligadas a radioterapia (Tabela 3 ). 


\section{Cuidados paliativos}

Neste tópico, os participantes descreveram a funcionalidade da assistência paliativa no município de Cabo Frio - RJ (Tabela 3).

Ao observar estes resultados, foi possível constatar que a maioria dos pacientes não recebe assistência paliativa como regulamentado, portanto evidencia que os profissionais envolvidos na assistência dessas pessoas apresentam carência de formação paliativista ${ }^{(13)}$.

\section{Discussão}

Apesar de o câncer afetar pessoas de todas as faixas etárias, torna-se uma evidência afirmar que indivíduos mais envelhecidos têm maior probabilidade de desenvolver a doença ${ }^{(4-14-15)}$. 0 fato de maior número do sexo feminino pode estar relacionado à maior sobrevida que este tem sobre o sexo masculino, esse fenômeno pode ser explicado pela maior taxa de mortes violentas que são mais comuns entre os homens durante a juventude, além de menor procura destes aos serviços de saúde ${ }^{(15)}$.

Este fenômeno não se diferencia na cidade de Cabo Frio - RJ, onde a população residente é de 95.396 mulheres, 4.565 a mais quando comparadas a população residente masculina(16). Mesmo assim, um estudo recente de incidência da doença no país aponta que os homens são mais acometidos pelo câncer que as mulheres devido a menor taxa de autocuidado e maior exposição a fatores de risco $^{(4-17)}$.
Sabe-se que pessoas com menores níveis de escolaridade podem estar mais pré-dispostas a fatores de risco em virtude da falta de informação e devido ao menor potencial aquisitivo, têm maior dificuldade para realizar consultas e exames de rotina, interferindo diretamente no desenvolvimento da doença e no diagnóstico $\operatorname{dela}^{(18)}$.

O CA de mama é o câncer mais incidente no mundo entre as mulheres, é a maior causa de mortes por câncer da população feminina. No Brasil, mais de 57 mil novos casos foram estimados para os anos de 2014/2015. Entre os principais fatores de risco estão: a idade, fatores reprodutivos, histórico familiar e sobrepeso. Já nos homens, o CA de próstata é o segundo mais incidente no mundo, e o tipo mais prevalente no país, perdendo apenas para o Tumor (TU) de pele não melanoma, com quase 69 mil novos casos estimados para os anos de 2014 e 2015 no Brasil, sendo o único fator de risco para desenvolvimento é a idade ${ }^{(4)}$.

O não funcionamento das políticas de saúde está relacionado à discordância entre as metas programadas e ações de práticas efetivas, ocorre por insuficiência de recursos ou limitações de conhecimento dos profissionais ${ }^{(19)}$.

O profissional de saúde tem papel de agente mobilizador, é fundamental que este tenha caráter proativo aproveitando os momentos junto ao paciente para orientá-lo de práticas preventivas e educativas para melhoria da qualidade de vida ${ }^{(19)}$.

Os serviços de atenção básica devem executar ações de promoção a saúde, pois realizam o acompanhamento ao longo da vida dos 
pacientes. A educação em saúde deve estar presente em atividades em grupo, que levam informações ao coletivo e também nas consultas individuais. É fundamental a disseminação do autocuidado e orientar quanto aos possíveis sinais de alerta do câncer(19).

A PNPCC constitui como princípio a promoção da saúde que visa identificar e intervir sobre os fatores causadores da doença, desenvolvendo estratégias de desenvolvimento intersetorial e de encargo do governo e da sociedade ${ }^{(5)}$

Esses fatores elevam o risco de indivíduos desenvolverem não só câncer, mas também várias doenças crônicas, desde que sejam expostos de forma cumulativa. A boa notícia é que indivíduos quando abandonam esses hábitos e adotam uma vida saudável, reduzem os riscos de desenvolvimento, ou seja, esses fatores são modificáveis, sendo assim, uma luta individual na batalha contra o câncer. A partir dessa descoberta, estima-se que $30 \%$ dos casos possam ser preveníeis $^{(16)}$.

A ampliação da rede saúde básica é um plano estratégico no combate contra as principais doenças crônicas, esse serviço cobre cerca de 60\% da população nacional, atuando em territórios específicos realizando ações de promoção, vigilância em saúde, prevenção, assistência e acompanhamento continuo ${ }^{(15)}$.

O controle do câncer está diretamente ligado a ações de promoção e prevenção. Fatores como tabagismo, consumo de álcool, ingesta de alimentos muito calóricos, sobrepeso, o impacto dos agrotóxicos, prática de relação sexual sem preservativo e exposição solar sem proteção são determinantes para o surgimento de novos casos. Uma educação permanente sobre hábitos de vida saudáveis é a base para prevenção, redução de danos relacionados à saúde e proteção da vida e, consequentemente, diminuição da incidência(4-5).

Para que ocorra a diminuição de casos, a prevenção e o controle da doença necessitam garantir o mesmo grau de atenção que os serviços assistenciais. Estratégias simples como o controle do tabagismo, incentivo de alimentação saudável, vacinação do Papiloma Vírus Humano (HPV) e hepatite, prática de atividades físicas, correspondem respectivamente à prevenção dos cânceres de pulmão, estômago, intestino, colo de útero, fígado, mama, próstata e outros ${ }^{(4)}$.

A prevenção primária contra o câncer está relacionada à adoção de hábitos de vida saudáveis e abandono ou diminuição da exposição aos fatores de risco, funciona como promoção da saúde. Já a prevenção secundária, é incorporada por tecnologias para rastreamento de determinados grupos e detecção precoce de novos $\operatorname{casos}^{(20)}$.

A prevenção é princípio da PNPCC, para haver controle da doença o objetivo é eliminar, reduzir e controlar fatores de risco de origem física, química e biológica, intervindo nos agentes causais da doença, além disso, a integração da ação de detecção precoce é imprescindível ${ }^{(5)}$.

São recomendações governamentais as ações de rastreamento e detecção precoce ${ }^{5}$. Rastreamento são exames feitos em pessoas assintomáticas pertencentes a uma população alvo, com intuito de identificar lesões precursoras ou cancerígenas em estado inicial. Já a detecção precoce utiliza sinais e sintomas apresentados 
pelo paciente associando a exposição aos fatores de risco, na tentativa de descobrir a doença mais cedo possível ${ }^{(20)}$.

A finalidade da detecção precoce é o rastreamento células pré-cancerígenas ou de CA ainda localizado em região de origem inicial, sem ocorrência de metástases. Com o alcance deste objetivo a relação entre efeito e custo é positiva e as possibilidades de cura e sobrevida do paciente se elevam, além de gerar melhor qualidade de vida ao doente ${ }^{16}$.

Através da identificação de fatores de risco e práticas autocuidado é alcançada uma detecção precoce e se inicia o tratamento oportuno, estas ações estão diretamente ligadas ao aumento no tempo de sobrevida e ao alcance da curabilidade ${ }^{(4-}$ 5).

Em decorrência da mudança demográfica que o país vem passando, associada ao progresso do processo industrial e urbano, avanços tecnológicos e científicos, o país é formado por uma população cada vez mais envelhecida com hábitos de vida que expõe a riscos, havendo maiores possibilidades de desenvolver doenças crônicas. O crescimento no número dos acometidos pelo câncer se deve a esse fenômeno ${ }^{(4)}$.

Se os números de novos casos continuarem aumentando é possível que a rede especializada de tratamento oncológico não consiga dar conta de realizar diagnósticos, tratamento e devido acompanhamento, em decorrência disso os óbitos serão mais frequentes $^{(4)}$.

No intuito de agilizar a realização dos exames diagnósticos os pacientes utilizam o próprio recurso financeiro. Devido ao alto custo destes exames, estes buscam o auxílio dos familiares para conseguir pagar, esta dificuldade compromete o orçamento desta família(20).

A detecção precoce é um dos princípios e diretrizes do cuidado integral no âmbito da PNPCC $^{(5)}$. Torna-se importante citar que o Governo Federal disponibiliza um repasse orçamentário para ações de média e alta complexidade, que assegura o diagnóstico e assistência dos pacientes usuários do sistema. No caso de prestadores de serviços privados, o processamento de autorizações gera o pagamento dos serviços oncológicos realizados ${ }^{(8)}$.

Vale lembrar que segundo a PNPCC, faz parte das Responsabilidades das Estruturas Operacionais das Redes de Atenção à Saúde, no Componente Atenção Básica implementar ações de diagnóstico precoce por meio da identificação de sinais e sintomas e encaminhar oportunamente a pessoa com suspeita de câncer para confirmação diagnóstica realizando um serviço de referência e contra referência ${ }^{(5)}$.

As UNACONs são hospitais que dispõe de condições técnicas, instalações físicas, equipamentos e recursos humanos apropriados para prestar assistência especializada de alta complexidade, tem o objetivo de traçar o diagnóstico definitivo e tratar os tipos de câncer que mais acometem a população brasileira. Já os CACONs têm todas as características de uma UNACON só se diferencia por tratar todos os tipos de câncer ${ }^{(6)}$.

O número de UNACONs e CACONs é calculado para atenderem, no mínimo 1.000 novos casos anuais. Porém a rede atenção 
oncológica parece não ser suficiente para atender todos os pacientes que necessitem de diagnóstico e tratamento. Um levantamento realizado pela Divisão de Apoio à Rede de Atenção Oncológica atualizado em 3 de junho de 2011, estimou que todo o território nacional necessitasse de mais 375 UNACONs e CACONs. Nesta época havia 264 serviços habilitados, sendo assim no total de 639 estabelecimentos para atender uma demanda estimada de 489.270 novos $\operatorname{casos}^{(8)}$.

Atualmente, a rede oncológica conta com 276 hospitais habilitados para prestar assistência a cerca de 576 mil novos $\operatorname{casos}^{(4-16)}$. Ou seja, analisando esses dados pode-se constatar que de 2011 até agora apenas 12 estabelecimentos foram credenciados e o número de acometidos pela doença é crescente, pontuando que ainda existe uma carência. Perante este fato, essa insuficiência de recursos não prejudica apenas o diagnóstico precoce, mas também o início de tratamento oportuno $^{(8)}$.

A promulgação da Portaria $n^{\circ} 876$, de 16 de maio de 2013 fixou como lei o prazo de 60 dias para início do primeiro tratamento, podendo ser esta cirurgia, radioterapia ou quimioterapia(20).

Os tumores malignos têm crescimento acelerado e são muito agressivos ao organismo, por causa da multiplicação rápida há grande possibilidade da formação de novos tumores além do local de origem, acometendo outros tecidos, iniciando um processo chamado metástase. A partir desse processo torna-se cada vez mais difícil a curabilidade, por isso a importância de um diagnóstico precoce e início de tratamento imediato $^{(4-20)}$.
Com a evolução dos tratamentos atuais alguns tipos de câncer metastático podem ter cura, mas a maioria não. Embora os tratamentos sejam disponibilizados a todos os pacientes com metástases, apenas uma minoria torna-se passível de cura. De modo geral, estes tratamentos têm o propósito controlar a evolução da doença ou amenizar os sintomas gerados pelas metástases, nestes casos a terapêutica pode contribuir para prolongar a sobrevida. Entretanto, a maioria das pessoas acometidas por metástases vem a óbito ${ }^{(13)}$.

Tal dificuldade relatada principalmente ao serviço de radioterapia relaciona-se ao déficit no número de aparelhos radioterápico, causando demora no início do tratamento ${ }^{(8-20)}$, além do incomodo de realizar o tratamento em outra cidade, dependendo do auxílio de familiares e amigos diante da debilidade causada pelo tratamento $^{(20)}$.

Um levantamento do Inca evidenciou uma carência de 135 unidades de atendimento ou equipamentos, mas segundo relatório do Tribunal de Contas da União publicado em 2011 esses números são ainda maiores, pois não foram computados os estabelecimentos que deixaram de prestar serviços para o SUS ${ }^{(8)}$.

A carência nos serviços de radioterapia não é relativa só a Região, mas sim a nível nacional. Atualmente, em todo o país existem 357 aparelhos radioterápicos, 269 destes atentem aos usuários SUS, mas seriam necessárias 680 máquinas para dar conta de toda a população(12|). Segundo um panorama realizado pela Sociedade Brasileira de Radioterapia, o serviço de radioterapia cobre cerca de $65,9 \%$ da demanda, e 
revela que quando os serviços são realizados não ocorrem de maneira tempestiva. O tempo médio de espera do diagnóstico ao início da radioterapia é de 113,4 dias, cerca de quatro meses, afetando diretamente a saúde do paciente ${ }^{(20)}$.

Relativo ao Estado do Rio de Janeiro, 27.816 novos casos precisaram de tratamento radioterápico. De acordo com a Portaria $n^{\circ} 741$ de 19 de dezembro de $2005^{(6)}$, cada aparelho de radioterapia poderá tratar 600 pacientes por ano, e no estado há 16 aparelhos, então 9.600 destes terão garantia de tratamento enquanto 18.216 não são atendidos(16).

Uma UNACON deve oferecer minimamente tratamentos quimioterápicos e cirúrgicos, porém a intervenção radioterápica deve ser referenciada formalmente a outra unidade que forneça o serviço, garantindo o direito de tratamento prescrito ao paciente ${ }^{(5)}$.

Quanto aos que necessitam de medicação de tratamento em casa (31\%), grande parte destes (73\%) dizem não comprar, pois são ofertadas pelo SUS (Tabela 3).

Dos participantes que utilizam terapia medicamentosa domiciliar, a maioria afirmou que recebem essa assistência pelo SUS, entretanto nota-se que nem todos usufruem o direito constitucional sobre a assistência terapêutica integral ${ }^{(11)}$.

O tratamento oncológico é de responsabilidade dos gestores municipais, estaduais e federais, o não fornecimento de medicações prescritas é uma falha no planejamento e acarreta processos judiciais ou financiamento próprio para suprir essa necessidade $\mathrm{e}^{(11-12)}$.
Os serviços de alta complexidade em oncologia credenciados pelo SUS funcionam também como Componentes do Sistema de Apoio prestando assistência farmacêutica indispensável à terapêutica, em regime domiciliar, ambulatorial e hospitalar de acordo com os termos da Lei $\mathrm{n}^{\circ} 12.401$, de 28 de abril de $2011^{(5-11)}$. Diante dos tipos de terapêuticas discutidas apresentam-se os cuidados paliativos que visam contribuir para o alivio do sofrimento e melhorar a qualidade de vida sem focar na curabilidade da doença ${ }^{(5-8-19)}$.

Em relação a Responsabilidade das Estruturas Operacionais das Redes de Atenção à Saúde da PNPCC, no Componente Atenção Básica, fica explicito a incumbência de realizar atendimento domiciliar e participar dos cuidados paliativos aos pacientes oncológicos de forma articulada com os demais serviços de saúde. Essas vistas deverão levar informações claras para a família e doente, garantir ênfase no controle da dor, proporcionar alivio do sofrimento e qualidade de vida com suporte e segurança(5).

A terapêutica ofertada ao paciente oncológico varia de acordo com tipo e estágio da doença, se dividem em tratamentos que objetivam a cura ou eliminação completa de células cancerosas, os que controlam a evolução da doença prolongando a sobrevida, e em casos em que não há mais possibilidade de curabilidade são iniciados os tratamentos paliativos que proporcionam o alivio dos sintomas causados pela doença $^{(20)}$.

Os cuidados paliativos, além de conter os sintomas físicos causados pela doença, compreendem ações psicoterapêuticas e espirituais do momento do diagnóstico 
confirmado até o óbito, esse apoio é ofertado não só para o paciente, mas também para a família. Para que esse serviço obtenha um atendimento satisfatório os profissionais prestadores de serviço devem receber uma educação permanente além das medidas psicoterápicas e estrutura espiritual. Esses cuidados devem ser prestados nas consultas ambulatoriais, em domicilio e nas internações de média e alta complexidade ${ }^{(13)}$.

Em face dos resultados obtidos, esta pesquisa foi de encontro ao objetivo e analisou a qualidade da política oncológica no município já citado, identificando algumas lacunas na assistência a partir do relato dos usuários do sistema.

\section{Conclusão}

Diante dos resultados obtidos, foi possível constatar há uma necessidade de ampliar a proposta de educação em saúde continuada, além do atendimento assistencial oncológico, para que possa cobrir a toda demanda de pacientes que necessitam de diagnóstico e tratamento, assim como assegura a política vigente.

Sabe-se que enquanto a promoção da saúde e a prevenção não obtiver o mesmo foco que os serviços assistenciais, secundário e terciário, o aumento nos números de morbimortalidade pelo câncer será crescente. Por esse motivo é importante que, além de aumentar a cobertura da rede básica, é primordial capacitar os profissionais envolvidos para que haja melhor aproveitamento e execução das políticas públicas de saúde.
Outro ponto importante para sugestão é levar mais conhecimento relativo à saúde para escolas, contribuindo para a formação de um novo ciclo de cidadãos que, quando educados desde cedo, levam a importância dos hábitos saudáveis para toda a vida e futuras gerações. Sendo assim, possivelmente, melhor será a aderência destas práticas e então melhor qualidade de vida terão no futuro.

Verificou-se uma falha no seguimento das linhas de cuidados, relativo ao encaminhamento oportuno dos pacientes com a suspeita da doença para confirmação. A demora na execução dos serviços de referência e contra referência para traçar o diagnóstico definitivo, os leva a custearem estes exames com seus ganhos, comprometendo parte se sua renda enquanto a oferta deveria ser gratuita.

Relativo ao atendimento assistencial oncológico, oferecido pelas UNACONs e CACONs, é perceptível que assim como no país, o município não consegue suprir a carência de toda clientela de forma equitativa e tempestiva. Devido ao aumento progressivo de acometidos pela doença, as estruturas de atendimento terciário deveriam crescer similarmente para que pudesse garantir os direitos da população, de atendimento integral e devida oferta terapêutica, assim como preconizado pela política oncológica.

Espera-se que este estudo possa colaborar para o aperfeiçoamento do processo de gestão da atenção oncológica do município, consequentemente melhorar a oferta de atendimento para que ocorra como o recomendado. 


\section{Referências}

1. Silva JVF, Silva EC, Rodrigues APRA, Miyazawa AP. A relação entre o envelhecimento populacional e as doenças crônicas não transmissíveis: Sério desafio de saúde Pública. Cadernos de Graduação: Ciências Biológicas e da Saúde, Maceió. 2015; 2(3):91-100.

2. Ceratti MK. O dilema entre debater o câncer ou a pobreza. El País. 2014 jul. 23; Internacional. [Internet]. [acesso 04 fev. 2018]. Disponível em: http://brasil.elpais.com/brasil/2014/07/22/intern acional/1406062733_078295.html.

3. Instituto Nacional de Câncer (INCA). O que é o câncer. [Internet]. [acesso 06 mar. 2018]. Disponível:

http://www1.inca.gov.br/conteudo_view.asp?id= 322.

4. Ministério da Saúde (BR). Instituto Nacional de Câncer José de Alencar. Estimativa 2014: Incidência de Câncer no Brasil. Rio de Janeiro (RJ); 2014.

5. Ministério da Saúde (BR). Portaria no 874, de 16 de maio de 2013. Institui a Política Nacional de Prevenção e Controle do Câncer na Rede de Atenção a Saúde das Pessoas com Doenças com Doenças Crônicas no âmbito do Sistema Único de Saúde. Diário Oficial da União. 2013; 17( Seção 1): 129-132.

6. Sitonio FT. Acesso ao tratamento oncológico no município de São Paulo: o câncer de mama como condição traçadora [dissertação]. São Paulo: Universidade de São Paulo, Faculdade de Saúde Pública; 2016.

7. A inauguração da Onkosol possibilita tratamento do Câncer em Cabo Frio. Jornal Tamoios. 2008; p.4. [Internet]. [acesso 17 abr. 2018].

Disponível: http://www.jornaltamoios.com.br/jornal-62/pag4.pdf.

8. Camargo RF. Avaliação da dose de radiação absorvida em exames radiológicos durante o planejamento radioterápico. 2014. 144 f. Dissertação (mestrado) - Universidade Estadual
Paulista Júlio de Mesquita Filho, Faculdade de Medicina de Botucatu, 2014.

9. Pereira JM. Manual de Metodologia da Pesquisa Científica. 4a ed. Rio de Janeiro: Atlas, 2016.

10. Ramos SS, Rodrigues LMS, Silva TASM, Balbino CM, Souza MMT, Silvino ZR. Conhecimentos, mitos e implicações para o cuidado de enfermagem no câncer de mama masculino. Rev Enf Atual. 2017; 83.

11. Malta DC, Silva MMA, Albuquerque GM, Lima $\mathrm{CM}$, Cavalcante $\mathrm{T}$, Jaime $\mathrm{PC}$, et al. The implementation of the priorities of the National Health Promotion Policy, an assessment, 20062014. Ciênc Saúde Coletiva 2014; 19(11): 4301-12.

12. Ramos AL, Silva DP, Machado GMO, Oliveira EM, Lima DS. Atuação do enfermeiro na estratégia de saúde da família na prevenção do câncer de colo de útero. Sanare. 2014; 13(1): 8491.

13. Brito FM, Costa ICP, Costa SFG, Andrade CG, Santos KFO, Francisco DP. Comunicação na iminência da morte: percepções e estratégia adotada para humanizar o cuidar em enfermagem. Escola Anna Nery Revista de Enfermagem. 2014; 18 (2):317-22.

14. Alves JED. Transição demográfica, transição da estrutura etária e envelhecimento. Revista Portal de Divulgação. 2014; 40:8-15.

15. Instituto Brasileiro de Geografia e Estatística (IBGE). Tábua Completa de Mortalidade para o Brasil nos períodos 2012-2013 e 1980-2013. Rio de Janeiro; 2014.

16. Instituto Brasileiro de Geografia e Estatística (IBGE). IBGE Cidades - Cabo Frio Síntese de informações. [Internet]. [acesso 23 mar. 2018]. Disponível: http://www.cidades.ibge.gov.br/xtras/temas.php ?lang $=\&$ codmun $=330070 \&$ idtema $=16 \&$ search=rio -de-janeiro|cabo-frio|sintese-das-informacoes.

17. Fundação do Câncer: com você pela vida. Falta de maquinas de radioterapia deixa mais de 18 mil pacientes com câncer sem tratamento no 
estado. 2014. [internet]. [acesso 15 mar. de 2018]. Disponivel: $\quad$ http://cancer.org.br/falta-demaquinas-de-radioterapia-deixa-mais-de-18-milpacientes-com-cancer-sem-tratamento-noestado/.

18. Pimentel J. Determinantes sociais da saúde. Apontamentos da PNS: pesquisa revela dados de saúde ligados aos hábitos de vida da população e aponta disparidades relacionadas à escolaridade e gênero. 2015 out. 13.

19. Buischi Petersen, C, Garcia de Lima, RA, Boemer, MR, Melo Rocha, SM. Necessidades de saúde e o cuidado de enfermagem. Revista Brasileira de Enfermagem 2016; 69(6):1236-39.

20. Batista DRR, Mattos $M$, Silva SF. Convivendo com o câncer: do diagnóstico ao tratamento. Rev Enferm UFSM. 2015; 5(3):499510. 